TRADITIONAL MEDICINE AND PHARMACOLOGY. ACHIEVEMENTS, INNOVATIONS, AND ALTERNATIVES

\title{
1.2 Processes of oxidative phosphorylation in tissues with actions of xenobiotics
}

Recently, the use of various organic nitrogen-containing compounds, including amines, in various industries and agriculture has increased significantly. These substances are used in the production of fertilizers, herbicides, pesticides, plastics. Aliphatic amines are found in large quantities in industrial air, water and soil, food and feed. Amines are also capable of being formed in animals, plants, and bacteria [31 - 35]. They perform a number of important physiological functions in the organism, namely, they act as hormones, mediators of nerve excitation, vitamins that are part of phosphatides, stimulate RNA synthesis and stabilize the DNA molecule of cell nuclei, and accelerate the growth and development of animals. The influence of biogenic high-molecular-weight amines on metabolic processes in tissues depends on their ability to form and modify hydrogen, covalent and ionic bonds with molecules of organic compounds. The aliphatic amines below have quite a different effect on metabolic processes in tissues [36].

An important scientific problem of modern biochemistry, which is of practical importance, is the study of the energy utilization processes of animal cells, which are based on numerous chemical reactions involving enzymes. However, in spite of the significant success achieved in studying the mechanisms of energy conversion in eukaryotes over the past 50 years, the problem of using the energy generated by the oxidation of organic substances in the cell in metabolic processes still remains incompletely elucidated today [37 - 39].

Eukaryotic cells are able to use the energy of chemical bonds of organic substances during their oxidation and deposit them as macroergic compounds, which include ATP, GTP, creatine phosphate, and some others. The above substances and especially ATP combine in the organism the two most important enzymatic systems providing for the conservation of chemical energy by oxidative phosphorylation reactions and its use in biosynthetic processes, mechanical muscle work, the maintenance of osmotic equilibrium in various cell compartments, etc. [40-42]. 
TRADITIONAL MEDICINE AND PHARMACOLOGY. ACHIEVEMENTS, INNOVATIONS, AND ALTERNATIVES

Numerous experimental data have so far confirmed the existence of a complex mechanism of a combination of the processes of oxidation of organic compounds and phosphorylation of ADP in the cell, which are inseparably linked to the formation, binding, and transport of electrons along the mitochondrial respiratory chain by means of specific carriers $[38,43,44]$.

Electrons and protons that were formed as a result of the action of NADP dependent dehydrogenases in CTC reactions, or the oxidative pentose phosphate cycle, enter the mitochondrial respiratory chain, where, moving with the help of specific carriers, cytochrome, they change their energy potential and are accepted by the $\mathrm{O} 2$ molecule. The components of the respiratory chain of eukaryotic mitochondria are localized mainly on the inner membrane and represent complex biological structures of protein (flavoproteins, FeS-proteins, cytochromes) and nonprotein (quinones) nature.[39, 43, 45]. Proton transfer is performed by flavoproteins and quinones, and electrons by FeS-proteins and cytochromes. The mechanism of proton and electron transfer along the respiratory chain is a complex process involving a number of numerous reactions, chief among which are those catalyzed by NADPdependent dehydrogenases and provide hydrogen stripping from the substrate and its transfer to NADP-H2 dehydrogenase, the starting carrier of the mitochondrial respiratory chain. FAD-dependent dehydrogenases have a similar function in the cell.

Hydrogen from these enzymes enters the respiratory chain at the level of quinones, which are capable of the reduction process by attaching two hydrogen atoms. One of the most important quinones that function on the pathway of hydrogen transfer from NADP-or FAD-dependent dehydrogenases to cytochrome is ubiquinone. Still, the main one in the respiratory chain of electron transfer is cytochromes. Four major cytochromes have been found in the mitochondrial respiratory chain, whose participation in electron transfer is determined by the following sequence: b, c, a, and a3 [38, 44]. According to their chemical origin, they are complex proteins containing heme as the prosthetic group. The molecular weight of cytochrome isolated from various plant and animal species ranges from 12.5 
TRADITIONAL MEDICINE AND PHARMACOLOGY. ACHIEVEMENTS, INNOVATIONS, AND ALTERNATIVES

(cytochrome from animal mitochondria) to $600.0 \mathrm{kDa}$ (the cytochrome complex aa3) [44].

It has been established that cytochromes differ among themselves not only by their absorption spectra and redox potentials, but also by their protein components, the nature of their side chains, and the way heme is attached to the protein. Cytochromes a and a3 function in a single complex as the enzyme cytochrome oxidase, which provides four electron transfer to oxygen, resulting in its activation followed by hydrogen addition and water formation. A peculiarity of the structure of cytochrome Aa3 molecule is the presence of not only iron ions but also copper ions in the molecule.

Cytochromes, or cellular pigments, are found in almost all living organisms, including the mitochondria of animal and plant cells, yeast, fungi, microsomes, chloroplasts, and E. coli, and their total number is about 30 different species. Spectral characteristics are used to characterize cytochromes and their functional properties. Cytochromes have the ability to accept or give up electrons, thus providing a certain sequence of reactions with energy release. It is this process that ensures the efficient use of energy by the cell without significant losses. The specific sequence of cytochrome placement in the respiratory chain of mitochondria is determined by their redox potential.

That is, the electron transporters in the mitochondrial respiratory chain are arranged according to their ability to be reduced, and each of the following cytochromes in the reduced state has a higher energy level than the previous one [43, 45].

A large group of substances has been established that can selectively block the process of electron transfer along the mitochondrial respiratory chain. These include barbiturates, a number of antibiotics, cyanides, azides, carbon monoxide, and others. For example, barbiturates block electron transfer from FAD-dependent dehydrogenases to $\mathrm{CoQ}$, the antibiotic actinomycin A blocks transfer from cytochrome $\mathrm{b}$ to $\mathrm{c}_{1}$, and cyanides, azides and $\mathrm{CO}$ block the above processes at the level of cytochrome aa 3 (cytochrome oxidase) [46]. 
TRADITIONAL MEDICINE AND PHARMACOLOGY. ACHIEVEMENTS, INNOVATIONS, AND ALTERNATIVES

The inhibition of electron transport by the above substances causes a decrease in the reduced forms of electron transporters located in the respiratory chain to the site of inhibitor action [47].

Studies of the effects of various toxicants on oxidative phosphorylation processes have been and are the subject of numerous experiments. On one hand, they are associated with the discovery of the underlying mechanisms of energy transformation in cell mitochondria, in particular the reactions of oxidative phosphorylation, transmembrane transport, and the ability to influence the difference between the electrochemical and electrical potential of the inner mitochondrial membrane, and on the other hand, with a search for effective medications to prevent the negative effect of xenobiotics $[48,49]$ and physical and chemical factors on the organism $[50,51]$.

It was found that chronic intoxication of white rats with sodium nitrate at a dose of $200 \mathrm{mg} / \mathrm{kg}$ body weight changes the functional state of the testes mitochondria, which is indicated by an increase in the rate of oxygen consumption and a dissociation of oxidative phosphorylation [52]. According to the authors, the latter is associated with an inhibitory effect of nitric oxide, a product of nitrite and nitrate ion metabolism, on the activity of respiratory chain enzymes, which leads to disruption of mitochondrial respiration and phosphorylation in the cells of the liver and kidneys of animals [53].

In addition, it is known that nitric oxide is capable of influencing the structure of the active center of aconitase, the iron-sulfur clusters of some cytochromes, and glyceraldehyde-3-phosphate dehydrogenase $[54,55]$. An inverse correlation between the level of iron-nitrosyl complexes and the rate of oxidation and phosphorylation processes has been established, indicating inhibition of the mitochondrial respiratory chain and decoupling of phosphorylation reactions under the influence of nitric oxide [52].

Administration of nitric oxide precursors L-arginine or NO-synthesis inhibitor $\mathrm{N} \omega$-nitro-L-arginine to rats also changes the intensity of oxidative phosphorylation reactions in mitochondria, the content of LPO products in blood, activity of amino 
TRADITIONAL MEDICINE AND PHARMACOLOGY. ACHIEVEMENTS, INNOVATIONS, AND ALTERNATIVES

acid reamination enzymes and succinate dehydrogenase with different animal resistance and their reaction to stress [56]. The inhibitory effect of nitric oxide with respect to the processes of oxidative phosphorylation in mitochondria, according to $[57,58]$, is associated with the ability of this cellular agent to bind to the iron heme of cytochrome c3 cytochrome c-oxidase, irreversibly interacting with the zylisosirkovimini centers of NADH-CoQ reductase and succinate-CoQ reductase, thus reducing the oxidation rate of substrates in mitochondria.

The ability of the cell and its individual structures, including mitochondria, to accumulate metals in most cases affects their functional state and changes the activity of enzymes and oxidative phosphorylation processes $[59,60]$. It has been shown that the character of the interaction of mitochondria with metals is determined by their physicochemical properties and primarily by the ability of metals to form bioligandams [61].

The addition of trichloromethoxycarbonyl cyanide-phenylhydrazone to the incubation medium increases the rate of mitochondrial respiration after their preincubation with $\mathrm{Ca}^{2+}, \mathrm{Sr}^{2+}$ and $\mathrm{Mn}^{2+}$ and does not affect the above processes after the interaction of these cell organelles with $\mathrm{Ca}^{2+}[62]$. The inhibition of proton-phasestimulated mitochondrial respiration by $\mathrm{Cd}^{2+}$ and $\mathrm{La}^{3+}$ was established, which indicates the ability of these ions to inhibit mitochondrial oxidation processes.

Of particular practical importance are studies of the influence of xenobiotics, substances that do not occur in nature but are synthetically produced, on energy processes in the mitochondria of cells of various organs and tissues closely related to their functional state.

Currently, more than 60,000 different types of xenobiotics are used in industry and agriculture, which in most cases are mutagens [63]. It was established that administration of different doses of mercazolinum to rats caused a decrease in the magnitude of ADP-stimulated respiration in the V3 Chance state and the phosphorylation rate in hepatocyte mitochondria [64]. Moreover, the above changes in mitochondria were observed both when the oxidation substrate succinate and $\alpha$ ketoglutarate were added to the incubation medium. In parallel with inhibition of 
TRADITIONAL MEDICINE AND PHARMACOLOGY. ACHIEVEMENTS, INNOVATIONS, AND ALTERNATIVES

tissue respiration processes, mercazoline activated LPO processes, in the authors' opinion, caused by inefficient utilization of the end product - malonic dialdehyde due to inhibition of redox processes in tissues. Based on the results obtained, the authors concluded that the action of mercazolinum as a mimic of secondary iodine deficiency, as well as thyroid gland dysfunction, is closely related to the functional state of liver mitochondria and lipoperoxidation processes that depend on the influence of hormones of this organ [64].

The obtained data once again confirm the conclusion that the liver plays the main role in the neutralization of compounds that are not intrinsic to the organism, and the toxic action of xenobiotics in the organism is based on modification of the structural and functional state of biological membranes, primarily the membranes of mitochondria and liver microsomes [65].

Administration of such a xenobiotic as paracetamol to rats of different ages caused a decrease in the rate of oxidative phosphorylation reactions and had no effect on oxygen consumption by liver mitochondria [63]. However, in animals of the experimental groups, a decrease in the content of cytochrome $b_{5}$ and 450 , an inhibition of the activity of aminopyrine-N-dimethylase and aniline-n-hydroxylase in the liver were found. The above changes in hemoprotein content and enzyme activity in the liver during the action of paracetamol were observed mainly in young animals and to a much lesser extent in older animals.

A special role in the antioxidant protection of the organism belongs to selenium, due to the unique ability of the latter to enter the structure of a number of enzymes, including glutathione reductase [66]. Selenium according to the electronic structure of the atom has a high reactivity. It is widespread in nature. It is found in soil and water [67], plants [68], fodder [69, 70, 71], animal and human tissues [72]. In the animal organism selenium enters with fodder, and in humans - with food products in the form of inorganic (selenite and selenites) and organic (selenomethionine and selenocysteine) forms [72]. It has been proved that the intensity of absorption of organic forms of selenium in the intestine is much higher than that of selenate and selenite $[73,74]$. Such elements as sulfur, copper, and 
TRADITIONAL MEDICINE AND PHARMACOLOGY. ACHIEVEMENTS, INNOVATIONS, AND ALTERNATIVES

cadmium inhibit selenium absorption in the intestine, reduce its content, and disrupt metabolism in tissues [75]. There are species, age, and dietary characteristics of selenium absorption [76, 77]. A study of the mechanism of selenium transport through the small intestinal mucosa has shown that it is a Na-dependent process [78], which is activated by $\alpha$-cysteine [79] and inhibited by sulfates, thiosulfates, molybdates and chromates [80], which compete with selenium for proteintransporters.

Selenium is highly depotable. It is found in red blood cells [81], liver [82], kidney [83], and sex glands [84], muscle and thyroid [85], pancreas, heart, lungs, and brain [86]. The selenium content in the above organs and tissues is influenced by the age of animals [87], their physiological state and hormonal status [83], and gender [88].

Selenium has antidote and radioprotective properties and reduces the negative impact of abiotic factors such as heavy metals on the body.

A decrease in tissue selenium content, in cadmium intoxication rats causes a decrease in the activity of antioxidant defense enzymes - glutathione peroxidase, SOD and catalase. This ultramicronutrient is able to improve the functional state of the kidneys in aluminum-cadmium intoxication in rats, as evidenced by a decrease in diuresis, and in blood plasma creatinine concentration, improved sodium and potassium transport in the kidneys, increased urine acidity and normalization of the blood ABB [88].

Selenium modifies the effect of ultraviolet irradiation in relation to the enzymes of the antioxidant system and LPO processes [71]. The oxidation of SH-groups of glutathione decreases, the activity of SOD and glutathione peroxidase increases, the level of hydroperoxides and malonic dialdehyde stabilizes.

Addition of sodium selenite to the diet of animals prevents the development of liver necrosis [89]. In a complex with some amino acids, such as cysteine, selenium in the form of selenocysteine enters the active center of glutathione peroxidase, which prevents the formation of methemoglobin in erythrocytes by cleaving hydrogen peroxide with glutathione [90]. 
Thus, it has been shown that one of the important factors preventing the negative effects of xenobiotics on the processes of oxidative phosphorylation in mitochondria and the processes of antioxidant protection is the trace element selenium. However, its role in the effect of low aliphatic amines on the body is practically unexplored.

In the experiment we studied the dynamics of the influence of ethyl and propylamine separately and in complex with sodium selenite on the intensity of oxidative phosphorylation processes in rat liver mitochondria. For this purpose, five groups of animals were formed - control and four research animals, 10 animals in each. The experiment was carried out according to the following scheme. The animals were transferred to a starvation diet at 12:00 p.m. before the administration of amines and sodium selenite. Rats of the first experimental group were injected with a special probe once per os $1 \mathrm{ml}$ of aqueous ethylamine solution at a dose of $80 \mathrm{mg} / \mathrm{kg}$ body weight. The animals of the second experimental group received $1 \mathrm{ml}$ of propylamine aqueous solution once per os at a dose of $76 \mathrm{mg} / \mathrm{kg}$ body weight. The animals of the third experimental group received ethylamine 60 minutes before loading in a dose of $80 \mathrm{mg} / \mathrm{kg}$ body weight, preliminarily administered once per os $1 \mathrm{ml}$ of sodium selenite aqueous solution in a dose of $80 \mathrm{mcg} / \mathrm{kg}$ body weight. The animals of the fourth experimental group also received $1 \mathrm{ml}$ of sodium selenite aqueous solution at a dose of $80 \mathrm{mcg} / \mathrm{kg}$ body weight 60 minutes before propylamine loading. Rats of the control group received $1 \mathrm{ml}$ of distilled water per os. The animals of control, experimental groups I and II were slaughtered by decapitation 10, 30, 60 and 120 minutes after amine injection, and those of groups III and IV after 120 minutes from the beginning of the experiment. Blood and liver samples were taken from the animals for the planned studies.

The dose of administration of aliphatic amines, ethyl and propylamine, to rats was set experimentally, taking into account the $\mathrm{LD}_{50}$, which is $400 \mathrm{mg} / \mathrm{kg}$ body weight of ethylamine and $370 \mathrm{mg} / \mathrm{kg}$ body weight of propylamine. We also took into account the minimum toxic dose of ethylamine for the rat body is $58-60 \mathrm{mg} / \mathrm{kg}$ body weight, and the minimum toxic dose for propylamine, which is $35-40 \mathrm{mg} / \mathrm{kg}$ body 
weight. The dose of sodium selenite administered to animals was calculated based on the recommendations for use [91].

Aliphatic amines, as shown in the previous section, affect not only physiological functions of the body, activity of lysosomal enzymes, phagocytic activity of neurophils, prothrombin formation, but can also change the activity of enzymes involved in redox reactions and, probably, oxidative phosphorylation processes in cell mitochondria.

Studies have shown that after giving rats per os water solution of ethylamine at a dose of $80 \mathrm{mg} / \mathrm{kg}$ body weight, which before this were on a 12-hour starvation diet, indicators of oxidative phosphorylation in the liver mitochondria after 10 minutes of exposure almost did not change (Table 1). It was found that the respiration rate of liver mitochondria after adding the oxidation substrate succinate $\left(\mathrm{V}_{2}\right)$ or the phosphorylation substrate $\operatorname{ADP}\left(\mathrm{V}_{3}\right)$ to the incubation medium, after depleting the phosphorylation substrate ADP $\left(\mathrm{V}_{4}\right)$ and after adding the phosphorylation inhibitor 2, 4-DNF (VDNF), as well as the rate of ADP phosphorylation $\left(\mathrm{V}_{\mathrm{F}}\right)$, the value of the phosphorylation coefficient $(\mathrm{ADP} / \mathrm{O})$ and the respiratory control $(\mathrm{RC})$ in the experimental group animals did not change compared with the control and corresponded to the conventional values (Table. 1).

However, in animals of the experimental group, which were decapitated in 30 minutes after injection of ethylamine solution, there were observed, although insignificant, changes in the indicators of oxidative phosphorylation in the liver mitochondria. Thus, it was found that in the animals of the experimental group compared with the control, the phosphorylation coefficient decreased by $16 \%$, the phosphorylation rate index by $33 \%$, and the respiratory control index by $19 \%$ (Table 1). Other indices, namely, mitochondrial respiration rate after addition of succinate, phosphorylation substrate - ADP before and after its depletion, and mitochondrial respiration inhibitor 2,4-DNF in the liver mitochondria of experimental group rats did not differ from similar indices in control group animals (Table 1). 
TRADITIONAL MEDICINE AND PHARMACOLOGY. ACHIEVEMENTS, INNOVATIONS, AND ALTERNATIVES

Table 1

Indices of oxidative phosphorylation in rat hepatocyte mitochondria under the action of ethylamine, $O_{2} / \mathrm{min} / \mathrm{mg}$ protein $\times 10^{-2}, \mathrm{Mm}, \mathrm{n}=6$

\begin{tabular}{|c|c|c|c|}
\hline \multirow{3}{*}{ Index } & \multicolumn{3}{|c|}{ Animal group } \\
\hline & \multirow[t]{2}{*}{ reference } & \multicolumn{2}{|c|}{$\begin{array}{l}\text { exploratory, min. after } \\
\text { ethylamine injection }\end{array}$} \\
\hline & & 10 & 30 \\
\hline $\mathrm{V}_{2}$ & $0,8 \pm 0,10$ & $0,9 \pm 0,10$ & $0,9 \pm 0,10$ \\
\hline $\mathrm{V}_{3}$ & $2,7 \pm 0,20$ & $3,3 \pm 0,20$ & $2,7 \pm 0,20$ \\
\hline $\mathrm{V}_{4}$ & $0,8 \pm 0,10$ & $0,9 \pm 0,10$ & $0,9 \pm 0,10$ \\
\hline $\mathrm{V}_{\mathrm{DNF}}$ & $2,1 \pm 0,20$ & $2,1 \pm 0,20$ & $2,0 \pm 0,20$ \\
\hline $\mathrm{ADF} / \mathrm{O}$ & $1,90 \pm 0,03$ & $1,80 \pm 0,05$ & $1,60 \pm 0,04 *$ \\
\hline $\mathrm{V}_{\mathrm{f}}, \mathrm{ATP} / \mathrm{min} / \mathrm{mg}$ protein & $0,060 \pm 0,002$ & $0,060 \pm 0,003$ & $0,040 \pm 0,02 *$ \\
\hline $\mathrm{BK}$ & $3,75 \pm 0,05$ & $3,67 \pm 0,10$ & $3,05 \pm 0,10^{*}$ \\
\hline
\end{tabular}

Note: * - marked a possible difference $(\mathrm{P} \leq 0,05)$ between the parameters of control and experiment.

During this time of ethylamine exposure of rats no changes in animal behavior were also observed. This indicates that the effect of ethylamine on physiological functions, intracellular liver metabolism including the processes of oxidative phosphorylation in mitochondria is related to its cumulation in tissues and appears, probably, somewhat later, which was confirmed by subsequent studies.

It was shown that 60 minutes after administration of an aqueous solution of ethylamine at a dose of $80 \mathrm{mg} / \mathrm{kg}$ body weight to experimental group animals per os, the most significant changes in the indicators of oxidative phosphorylation in mitochondria of hepatocytes were observed (Table 2).

It was established that in the liver of rats 60 min after ethylamine injection in comparison with the analogous indices in the control group animals not only phosphorylation rate of ADP and respiratory control coefficient decreased by 48,1 
TRADITIONAL MEDICINE AND PHARMACOLOGY. ACHIEVEMENTS, INNOVATIONS, AND ALTERNATIVES

and $36 \%$ respectively, but mitochondrial respiration rate also decreased by $33 \%$ after introducing into the incubation medium the decoupling agent 2,4-DNF as the factor inhibiting the processes of oxidation and phosphorylation in the mitochondrial respiratory chain (Table 2$)$.

It is also known that the effect of most disruptors of oxidative phosphorylation is associated with a decrease in the proton resistance of the membrane lipid bilayer.

It is possible to assume that inhibitory effect of ethylamine on the processes of oxidative phosphorylation in mitochondria of hepatocytes as evidenced by the decrease of $\mathrm{O} 2$ consumption and phosphorylation of ADP caused by the change of mitochondrial membrane $\mathrm{pH}$ gradient, as $\mathrm{H}+$ transport through the mitochondria membrane is connected with ATP synthesis and closely connected with proton movement through ion channels formed by ATP-synthetase enzyme.

A change in $\mathrm{pH}$ on the outer and inner sides of the mitochondrial membrane also inhibits electron transfer through the above structures, which probably affects cytochrome activity that forms the mitochondrial respiratory chain and decreases the rate of proton transfer to oxygen.

Thus, a decrease in the energetic function of rat hepatocyte mitochondria after ethylamine injection was established, which is closely related to the duration of the toxicant.

Table 2

Indices of oxidative phosphorylation in rat hepatocyte mitochondria under the action of ethylamine, $\mathrm{O} 2 / \mathrm{min} / \mathrm{mg}$ protein $\mathrm{x10-2,Mm,} \mathrm{n}=6$

\begin{tabular}{|c|c|c|c|}
\hline \multirow{3}{*}{ Index } & \multicolumn{3}{|c|}{ Animal group } \\
\hline & \multirow[t]{2}{*}{ reference } & \multicolumn{2}{|c|}{$\begin{array}{l}\text { exploratory, min. after } \\
\text { ethylamine injection }\end{array}$} \\
\hline & & 60 & 120 \\
\hline $\mathrm{V}_{2}$ & $0,8 \pm 0,10$ & $0,8 \pm 0,10$ & $0,9 \pm 0,10$ \\
\hline $\mathrm{V}_{3}$ & $2,7 \pm 0,20$ & $1,8 \pm 0,20 *$ & $2,9 \pm 0,20$ \\
\hline $\mathrm{V}_{4}$ & $0,8 \pm 0,10$ & $0,8 \pm 0,10$ & $0,8 \pm 0,10$ \\
\hline
\end{tabular}


TRADITIONAL MEDICINE AND PHARMACOLOGY. ACHIEVEMENTS, INNOVATIONS, AND ALTERNATIVES

\begin{tabular}{|l|c|c|c|}
\hline $\mathrm{V}_{\mathrm{DNF}}$ & $2,1 \pm 0,20$ & $1,4 \pm 0,20^{*}$ & $1,6 \pm 0,20$ \\
\hline $\mathrm{ADF} / \mathrm{O}$ & $1,90 \pm 0,03$ & $1,54 \pm 0,04^{*}$ & $1,70 \pm 0,05$ \\
\hline $\mathrm{V}_{\mathrm{f}}, \mathrm{ATP} / \mathrm{min} / \mathrm{mg}$ protein & $0,060 \pm 0,002$ & $0,031 \pm 0,002^{*}$ & $0,054 \pm 0,002$ \\
\hline $\mathrm{BK}$ & $3,75 \pm 0,05$ & $2,40 \pm 0,08^{*}$ & $3,45 \pm 0,10$ \\
\hline
\end{tabular}

Note: ${ }^{*}$ - marked a possible difference $(\mathrm{P} \leq 0,05)$ between the parameters of control and experiment.

It was shown that the intensity of oxidative phosphorylation in liver mitochondria decreased with prolongation of ethylamine action in animals of experimental group (Tables 1,2). Thus, in rats $60 \mathrm{~min}$ after ethylamine injection in comparison with analogous indices in animals $30 \mathrm{~min}$ after introduction of the toxicant, mitochondrial respiration rate after adding the phosphorylation substrate ADP in incubation medium decreased by $33 \%$, after adding 2,4-DNF - by $30 \%$, phosphorylation rate - by $22.5 \%$, respiratory control coefficient - by $21.3 \%$, whereas such indices as mitochondria respiration rate after adding succinate $\left(\mathrm{V}_{2}\right)$ and after depleting ADP $\left(\mathrm{V}_{4}\right)$ to incubation medium and phosphorylation coefficient (ADP/O) did not change. The obtained data indicate that toxic effects of ethylamine in animals depend not only on the dose and manner of introduction of this toxicant into the organism, but also on the time of exposure and activity of its detoxification mechanisms which are closely connected with the action of monoamine oxygenase and the rate of utilization of ammonia nitrogen by the liver.

Confirmation of the previous assumption the results of studies of the indicators of oxidative phosphorylation in the liver mitochondria of rats in 2:00 after ethylamine injection (Table 2). It was shown that the rate of mitochondrial respiration after 2:00 experiment after addition of the phosphorylation substrate ADP to the incubation medium in the experimental group rats increased by $61 \%$, the phosphorylation rate by $74 \%$, and the respiratory control ratio by $43.7 \%$, while the mitochondrial respiration rate after addition of succinate, depletion of ADP, addition of the disrupter-2,4 DNF, and the phosphorylation ratio did not change compared with similar indices in animals in the experimental group one hour after ethylamine (Table 2). 
Comparing the results obtained with similar indices in animals of the control group, it should be stated that 2:00 after the beginning of the action of ethylamine in the body of animals at its oral administration at a dose of $80 \mathrm{mg} / \mathrm{kg}$ body weight its negative effect of this toxicant on the indicators of oxidative phosphorylation in the liver mitochondria sharply decreased, and the indicators that characterize it corresponded to the norm. This may indicate quite active mechanisms of ethylamine conversion in the liver of animals, associated primarily, as noted above, with the action of the enzyme monoamine oxygenase and cytochrome $\mathrm{P}_{450}$.

It was found that 2:00 after administration of ethylamine to rats, the rate of mitochondrial respiration after addition of the phosphorylation substrate ADP to the incubation medium, its depletion, the addition of disrupter - 2.4 DNF, the rate of ADP phosphorylation, the respiratory control coefficient, and the phosphorylation coefficient were at the level of the control group (Tables 1,2).

Studying the effects of another aliphatic amine, propylamine, on rats, we found similar changes in the character of oxidative phosphorylation processes in liver mitochondria, and for the effects of ethylamine on animals (Tables 3, 4). However, the toxic effects of propylamine on the rat organism were more significant than those of ethylamine, as evidenced by the indicators of oxidative phosphorylation in mitochondria given below (Tables 3, 4).

It was shown that, as in the case of ethylamine, after per os injection of propylamine at a dose of $76 \mathrm{mg} / \mathrm{kg}$ body weight into rats after $10 \mathrm{~min}$ of the experiment, the indicators of oxidative phosphorylation in the liver mitochondria of rats did not change as compared with the control. Thus, the rate of mitochondrial respiration after adding the oxidation substrate succinate, the phosphorylation substrate ADP, to the incubation medium and after its depletion, and after adding the disruptor of tissue respiration and phosphorylation, 2,4-DNF, the phosphorylation rate, the respiratory control factor, and the phosphorylation factor in the liver mitochondria of the experimental group rats remained at the control level (Table 3).

However, 30 minutes after administration of propylamine to the rats of the study group, a $16 \%$ decrease in the phosphorylation factor, a $29 \%$ decrease in the 
TRADITIONAL MEDICINE AND PHARMACOLOGY. ACHIEVEMENTS, INNOVATIONS, AND ALTERNATIVES

phosphorylation rate, and an $18 \%$ decrease in the respiratory control factor were observed in the mitochondria of hepatocytes compared with the same indices in the control group animals (Table 3). Comparing these data with the analogous indices in the animals of the experimental group in 10 minutes after propylamine injection we should state that after 30 minutes of the experiment the mitochondria respiration rate after adding to the incubation medium of ADP phosphorylation substrate decreased by $19 \%$, the ADP phosphorylation rate - by $30 \%$, the respiration control coefficient - by $23 \%$ and the phosphorylation factor - by $11 \%$. At the same time, such indices as mitochondrial respiration rate after adding succinate, ADP phosphorylation substrate before and after its depletion, tissue respiration decoupler and 2,4-DNF phosphorylation rate practically did not change in the experimental group rats after 30 min of experiment as compared with the control.

Table 3

Indices of oxidative phosphorylation in rat hepatocyte mitochondria under the action of propylamine, $\mathrm{O}_{2} / \mathrm{min} / \mathrm{mg}$ protein $\times 10^{-2}, \mathrm{Mm}, \mathrm{n}=6$

\begin{tabular}{|l|c|c|c|}
\hline \multirow{2}{*}{ Index } & \multicolumn{3}{|c|}{ animal group } \\
\cline { 2 - 4 } & \multirow{2}{*}{ Reference } & \multicolumn{2}{|c|}{$\begin{array}{c}\text { exploratory, min. after } \\
\text { ethylamine injection }\end{array}$} \\
\cline { 2 - 4 } & & 10 & 30 \\
\hline $\mathrm{V}_{2}$ & $0,8 \pm 0,1$ & $0,9 \pm 0,1$ & $0,8 \pm 0,1$ \\
\hline $\mathrm{V}_{3}$ & $2,7 \pm 0,2$ & $3,1 \pm 0,2$ & $2,5 \pm 0,2$ \\
\hline $\mathrm{V}_{4}$ & $0,8 \pm 0,1$ & $0,8 \pm 0,1$ & $0,9 \pm 0,1$ \\
\hline $\mathrm{V}_{\mathrm{DNF}}$ & $2,1 \pm 0,2$ & $1,8 \pm 0,2$ & $1,7 \pm 0,2$ \\
\hline $\mathrm{ADF} / \mathrm{O}$ & $1,90 \pm 0,03$ & $1,78 \pm 0,06$ & $1,58 \pm 0,05^{*}$ \\
\hline $\mathrm{V}_{\mathrm{f}}, \mathrm{ATP} / \mathrm{min} / \mathrm{mg}$ protein & $0,060 \pm 0,002$ & $0,056 \pm 0,003$ & $0,039 \pm 0,002^{*}$ \\
\hline $\mathrm{BK}$ & $3,75 \pm 0,05$ & $3,56 \pm 0,08$ & $2,72 \pm 0,07^{*}$ \\
\hline
\end{tabular}

Note: ${ }^{*}$ - marked a possible difference $(\mathrm{P} \leq 0,05)$ between the parameters of control and experiment 
As in the case of ethylamine, the most significant effect of propylamine on the processes of oxidative phosphorylation in hepatocyte mitochondria was registered in rats after 60 minutes of the experiment. Only such indices as mitochondrial respiration rate after introduction of succinate into the incubation medium and after depletion of the phosphorylation substrate did not change in animals of the experimental group as compared with the control. Whereas mitochondrial respiration rate after adding ADP phosphorylation substrate to incubation medium, after adding 2,4-DNP phosphorylation disruptor and oxidative phosphorylation disruptor, phosphorylation rate, respiratory control ratio and phosphorylation ratio in $60 \mathrm{~min}$ after adding propylamine to rats decreased by 33; 33; 16.5 and $47 \%$ respectively as compared with controls (Table 4).

Propylamine, like ethylamine, had the most pronounced effect on oxidative phosphorylation in rat liver mitochondria 60 minutes after administration. It was shown that in comparison with the data obtained in the experiment after 30-min exposure of rats to propylamine, the mitochondria respiration rate in $60 \mathrm{~min}$ of experiment after adding succinate to the incubation medium was decreased by $44 \%$ and the rate of tissue respiration and oxidative phosphorylation disruptor 2,4-DNF - by $29 \%$ as compared with control. The rate of ADP phosphorylation, and the respiratory control ratio in liver mitochondria 60 minutes after administration of propylamine to rats decreased compared with similar data in animals after 30 minutes of experiment by 49 and $16.5 \%$, respectively (Tables 3, 4).

The obtained data indicate that despite the insignificant amount and single administration to rats, propylamine inhibits the processes of oxidative phosphorylation in the liver mitochondria. However, in this dose, the effect of propylamine is short-term and liver cells are able to convert the toxic compound into other metabolites within two hours, as evidenced by the stability of the indicators of oxidative phosphorylation in hepatocyte mitochondria and the activity of antioxidant protection enzymes in tissues (Tables 7-9).

It was shown that 2: 00 after initiation of administration of propylamine to rats of the study group, such indicators of oxidative phosphorylation as mitochondrial respiration rate after addition of phosphorylation substrate ADP, after its depletion, introduction of tissue respiration and oxidative phosphorylation disruptor 2,4-DNF, phosphorylation rate, 
TRADITIONAL MEDICINE AND PHARMACOLOGY. ACHIEVEMENTS, INNOVATIONS, AND ALTERNATIVES

respiration control and phosphorylation ratio were higher by 12.1, respectively; 50; 50; $50 ; 12.7 ; 16.5$ and $52 \%$ compared with the same indices in the experimental group rats after 60 minutes of experiment and corresponded to the corresponding values of these indices in the control group animals (Tables. 3, 4).

Thus, it was found that per os administration to rats of ethyl- or propylamine in doses of 80 or $76 \mathrm{mg} / \mathrm{kg}$ body weight, respectively, significantly inhibited the rate of ADP phosphorylation in mitochondria and, to a much lesser extent, the rate of succinate oxidation. Moreover, the effect of propylamine on the above-mentioned processes in rat liver mitochondria was significantly more pronounced than that of ethylamine. The latter is probably due to the structure of the molecule of these compounds, some differences in the physicochemical properties of the solutions of these substances, peculiarities of the mechanisms of their deactivation in the liver and the rate of utilization of the cleavage products.

Tables 4

Indices of oxidative phosphorylation in rat hepatocyte mitochondria under the action of propylamine, $\mathrm{O}_{2} / \mathrm{min} / \mathrm{mg}$ protein $\times 10^{-2}, \mathrm{Mm}, \mathrm{n}=6$

\begin{tabular}{|c|c|c|c|}
\hline \multirow{3}{*}{ Index } & \multicolumn{3}{|c|}{ Animal group } \\
\hline & \multirow[t]{2}{*}{ reference } & \multicolumn{2}{|c|}{$\begin{array}{l}\text { exploratory, min. after } \\
\text { ethylamine injection }\end{array}$} \\
\hline & & 60 & 120 \\
\hline $\mathrm{V}_{2}$ & $0,8 \pm 0,1$ & $0,8 \pm 0,1$ & $1,0 \pm 0,1$ \\
\hline $\mathrm{V}_{3}$ & $2,7 \pm 0,2$ & $1,4 \pm 0,2 *$ & $3,1 \pm 0,2$ \\
\hline $\mathrm{V}_{4}$ & $0,8 \pm 0,1$ & $0,6 \pm 0,1$ & $0,9 \pm 0,1$ \\
\hline $\mathrm{V}_{\mathrm{DNF}}$ & $2,1 \pm 0,2$ & $1,2 \pm 0,1^{*}$ & $1,8 \pm 0,2$ \\
\hline $\mathrm{ADF} / \mathrm{O}$ & $1,90 \pm 0,03$ & $1,49 \pm 0,06^{*}$ & $1,68 \pm 0,04 *$ \\
\hline $\mathrm{V}_{\mathrm{f}}, \mathrm{ATP} / \mathrm{min} / \mathrm{mg}$ protein & $0,060 \pm 0,002$ & $0,020 \pm 0,002 *$ & $0,053 \pm 0,003$ \\
\hline $\mathrm{BK}$ & $3,75 \pm 0,05$ & $2,27 \pm 0,08^{*}$ & $3,47 \pm 0,09$ \\
\hline
\end{tabular}

Note: * - marked a possible difference $(\mathrm{P} \leq 0,05)$ between the parameters of control and experiment 
TRADITIONAL MEDICINE AND PHARMACOLOGY. ACHIEVEMENTS, INNOVATIONS, AND ALTERNATIVES

Thus, the rate of liver mitochondrial respiration after adding ADP to the incubation medium 60 minutes after administration of propylamine to the animals compared with the control decreased 1.92-fold, and ethylamine - 1.5-fold, after adding the oxidative phosphorylation and tissue respiration disrupter 2,4-DNF - 1.75 and 1.5 times, respectively, the phosphorylation rate -3.0 and 1.93 times, the respiratory control rate -1.65 and 1.56 times, and the phosphorylation rate -1.27 and 1.23 times. The rate of mitochondrial respiration after depletion of ADP, 60 minutes after administration of propylamine to the animals, decreased 1.33-fold, and ethylamine without changes compared with the control, as well as the rate of mitochondrial respiration after adding the oxidation substrate - succinate to the incubation medium in the first and second cases (Tables 1,4).

Thus, the results obtained support the preliminary conclusion that the inhibitory effect of the lower aliphatic amines ethyl- and propylamine on oxidative phosphorylation in mitochondria is associated primarily with changes in the mitochondrial membrane $\mathrm{pH}$ gradient and the rate of electron transport along the mitochondrial respiratory chain. Since $\mathrm{H}+$ transport from the mitochondrial membrane is conjugated with ATP synthesis, the decrease in ADP phosphorylation rate and phosphorylation and respiratory control ratios closely related to the decrease in mitochondrial respiration rate upon introduction of the ADP phosphorylation substrate into the incubation medium can be explained by the effect of amines on $\mathrm{pH}$ of the intracellular medium.

The degree of influence of aliphatic amines on the processes of oxidative phosphorylation in rat liver mitochondria also depends on the duration of toxin action. The most significant effect of amines on the above processes is observed 30 and 60 minutes after ethylamine injection and 30,60 and 120 minutes after propylamine injection. Noteworthy is also the fact that ethyl and propylamine at doses of 80 and 76 $\mathrm{mg} / \mathrm{kg}$ body weight, respectively, show a greater inhibitory effect on ADP phosphorylation processes than on succinate oxidation.

Direct effect of ethyl- and propylamine on oxidative phosphorylation processes in liver mitochondria under in vivo conditions indicates that aliphatic amines have a 
TRADITIONAL MEDICINE AND PHARMACOLOGY. ACHIEVEMENTS, INNOVATIONS, AND ALTERNATIVES

direct effect on mitochondria and decrease their functional activity resulting in deficiency of macroergic compounds in cells.

The indicated conclusion is confirmed by the results of studies effects of ethyland propylamine on oxidative phosphorylation in intact mitochondria of hepatocytes.

Tables 5

Indices of oxidative phosphorylation in rat hepatocyte mitochondria under the action of ethyl- and propylamine (in vitro), $\mathrm{O}_{2} / \mathrm{min} / \mathrm{mg}$ protein $\times 10^{-2}, \mathrm{Mm}$, $\mathrm{n}=6$

\begin{tabular}{|l|c|c|c|}
\hline \multirow{2}{*}{ Index } & \multicolumn{3}{|c|}{ animal group } \\
\cline { 2 - 4 } & \multirow{2}{*}{ Reference } & \multicolumn{2}{|c|}{ exploratory } \\
\cline { 3 - 4 } & & ethylamine & propylamine \\
\hline $\mathrm{V}_{2}$ & $0,8 \pm 0,1$ & $0,6 \pm 0,1$ & $0,7 \pm 0,1$ \\
\hline $\mathrm{V}_{3}$ & $2,7 \pm 0,2$ & $1,1 \pm 0,1^{*}$ & $1,0 \pm 0,2^{*}$ \\
\hline $\mathrm{V}_{4}$ & $0,8 \pm 0,1$ & $0,4 \pm 0,1^{*}$ & $0,4 \pm 0,1^{*}$ \\
\hline $\mathrm{V}_{\mathrm{DNF}}$ & $2,1 \pm 0,2$ & $0,9 \pm 0,2^{*}$ & $1,0 \pm 0,1^{*}$ \\
\hline $\mathrm{ADF} / \mathrm{O}$ & $1,90 \pm 0,03$ & $1,85 \pm 0,03$ & $1,85 \pm 0,03$ \\
\hline $\mathrm{V}_{\mathrm{f}}, \mathrm{ATP} / \mathrm{min} / \mathrm{mg}$ protein & $0,060 \pm 0,002$ & $0,023 \pm 0,002^{*}$ & $0,019 \pm 0,002^{*}$ \\
\hline $\mathrm{BK}$ & $3,75 \pm 0,05$ & $2,4 \pm 0,08^{*}$ & $2,21 \pm 0,10^{*}$ \\
\hline
\end{tabular}

Note: * marked a possible difference $(\mathrm{P} \leq 0,05)$ between the parameters of control and experiment

In experiments performed in vitro the inhibitory effect of ethyl- and propylamine was also found on both individual links of the mitochondrial respiratory chain and the processes of ATP synthesis from ADP and PH.

It was shown that after adding ethylamine to the incubation medium in a dose of $11 \mathrm{mg}$ per sample, the rate of mitochondrial respiration after adding the substrate of ADP phosphorylation was 2.45 and after depletion of ADP - 2 times, after adding the uncoupling agent of oxidative phosphorylation 2,4-DNF - 2.33, the rate of phosphorylation - 2.61 and the respiratory control factor - 1.56 times in comparison with 
control. Ethylamine had no effect under the experimental conditions on mitochondrial respiration rate when the oxidation substrate succinate was added to the incubation medium and on the phosphorylation coefficient compared with the same indices in the mitochondria of the control group (Table 5). It should be noted that the effect of ethylamine on oxidative phosphorylation in liver mitochondria introduced directly into the incubation medium was significantly stronger than when this toxicant was administered to animals per os. This is evidenced by the results of the studies shown in Tables 1, 2, and 5. Thus, both in animal experiments and in in vitro studies, a significant effect of ethylamine on oxidative phosphorylation in the liver mitochondria was established, confirming the view that the aliphatic amines act, probably, in changing the rate of ion flow through the ion channels of the mitochondrial membrane. This causes a change in the charge of the latter, thus reducing the activity of ATP synthetase. Ethylamine affects the rate of mitochondrial respiration to a much lesser extent after adding the oxidation substrate succinate.

Similar changes in the character of oxidative phosphorylation parameters in intact mitochondria were obtained when propylamine was added to the incubation medium (Table 5).

It was shown that after adding propylamine aqueous solution in a dose of $7 \mathrm{mg}$ per sample set experimentally to incubation medium, the respiration rate of intact mitochondria after adding ADP to incubation medium decreased 2.7-fold, 2.0-fold after its depletion, 2.1-fold after addition of the oxidative phosphorylation and tissue respiration disruptor 2,4-DNF, reduces the phosphorylation rate of ADP by 3.25 -fold and the respiratory control ratio by 1.7 -fold compared to the control. Whereas mitochondrial respiration rate after succinate addition and phosphorylation coefficient did not change compared with the same parameters in the control (Table 5).

The data obtained confirm the previously expressed opinion that the inhibitory effect of lower aliphatic amines is associated primarily with the effect on ADP phosphorylation processes, with a decrease in macroergic compounds in the cell and to a lesser extent with substrate oxidation. 
Propylamine, in spite of the fact that it was added to the incubation medium at a dose 1.5 times lower than ethylamine, caused almost identical changes in oxidative phosphorylation parameters in mitochondria. Thus, mitochondrial respiration rate after addition of ADP to the incubation medium, its depletion, introduction of the oxidative phosphorylation disruptor and tissue respiration 2,4-DNF, as well as phosphorylation rate and respiratory control ratio remained low after addition of propylamine as compared with control. The latter indicates a significantly higher toxicity for the animal organism of propylamine and its more significant effect on the phosphorylation processes in the liver mitochondria.

Thus, based on the results of the studies, we can conclude that the toxic effect of lower aliphatic amines - ethyl and propylamine is associated with the effect directly on the mitochondrial membrane, processes of ADP phosphorylation, formation of ATP and other macroergic compounds. Since in in vitro experiments with the addition of ethyland propylamine to the incubation medium where the oxidative phosphorylation disruptor 2,4-DNF was previously added, a further significant decrease in the mitochondrial respiration rate (VDNF) was observed, we can conclude that the mechanism of the negative effect of low aliphatic amines on oxidative phosphorylation is related to their conversion in the cytoplasm into a proton, penetration of the latter through ion channels into the mitochondria, depolarization as a consequence of the mitochondrial membrane leads to a decline in ADP phosphorylation processes.

One way to prevent the negative effects of the aliphatic amines ethyl and propylamine on the processes of oxidative phosphorylation in the mitochondria, in addition to the dose and duration of action of these toxicants on the body can be substances that prevent penetration of amines into the cell cytoplasm, promoted their conversion into non-toxic metabolites, the formation of protons with amines or the formation of their complexes with organic acids, which inhibited their transport through the ion channels of mitochondrial membranes.

As shown by studies, sodium selenite can be one such substance, due to the unique property of selenium to exhibit antioxidant effect in tissues and to be a part of the glutathione peroxidase molecule. 
Studies have established that 60 minutes prior to loading rats with ethyl or propylamine, giving them per os an aqueous solution of sodium selenite at a dose of 80 $\mathrm{mcg} / \mathrm{kg}$ body weight prevents the negative effects of these compounds on the processes of oxidative phosphorylation in the liver mitochondria (Table 6).

It was shown that the respiration rate of liver mitochondria of rats treated with sodium selenite and then ethylamine after adding succinate, phosphorylation substrate ADP to the incubation medium and after its depletion, the addition of the oxidative phosphorylation and tissue respiration disruptor 2,4-DNF, and the phosphorylation rate, phosphorylation factor, and respiration control factor were not different from those of control group animals. The results obtained indicate that sodium selenite administered to the animals in advance as an aqueous solution per os shows a protective effect in relation to ethylamine, which is probably due to the high antioxidant properties of this trace element and its role in amine metabolism processes in the cell through participation in the glutathione peroxidase active center structure.

This conclusion is also confirmed by the results of studies on the effect of propylamine on the processes of oxidative phosphorylation in the liver mitochondria and on the activity of several liver and blood enzymes of rats which were previously injected with sodium selenite solution (Tables 6-8).

Tables 6

Indices of oxidative phosphorylation in rat liver mitochondria under the action of sodium selenite and ethyl- and propylamine, $\mathrm{O}_{2} / \mathrm{min} / \mathrm{mg}$ protein $\times 10^{-}$

${ }^{2}, \mathrm{Mm}, \mathrm{n}=\mathbf{6}$

\begin{tabular}{|l|c|c|c|}
\hline \multirow{2}{*}{ Index } & \multicolumn{3}{|c|}{ Animal group } \\
\cline { 2 - 4 } & reference & \multicolumn{2}{|c|}{ exploratory } \\
\cline { 3 - 4 } & $\begin{array}{c}\text { sodium selenite } \\
+ \text { ethylamine }\end{array}$ & $\begin{array}{c}\text { sodium selenite } \\
+ \text { propylamine }\end{array}$ \\
\hline $\mathrm{V}_{2}$ & $0,8 \pm 0,1$ & $0,9 \pm 0,1$ & $0,9 \pm 0,1$ \\
\hline $\mathrm{V}_{3}$ & $2,7 \pm 0,2$ & $3,0 \pm 0,2$ & $3,0 \pm 0,2$ \\
\hline
\end{tabular}


TRADITIONAL MEDICINE AND PHARMACOLOGY. ACHIEVEMENTS, INNOVATIONS, AND ALTERNATIVES

\begin{tabular}{|l|c|c|c|}
\hline $\mathrm{V}_{4}$ & $0,8 \pm 0,1$ & $0,8 \pm 0,1$ & $0,9 \pm 0,1$ \\
\hline $\mathrm{V}_{\mathrm{DNF}}$ & $2,1 \pm 0,2$ & $1,7 \pm 0,2$ & $1,8 \pm 0,3$ \\
\hline $\mathrm{ADF} / \mathrm{O}$ & $1,90 \pm 0,03$ & $1,78 \pm 0,05$ & $1,76 \pm 0,05$ \\
\hline $\mathrm{V}_{\mathrm{f}}, \mathrm{ATP} / \mathrm{min} / \mathrm{mg}$ protein & $0,060 \pm 0,002$ & $0,050 \pm 0,002$ & $0,054 \pm 0,002$ \\
\hline $\mathrm{BK}$ & $3,75 \pm 0,05$ & $3,54 \pm 0,1$ & $3,43 \pm 0,08$ \\
\hline
\end{tabular}

It was shown that 60 minutes before propylamine injection, application of sodium selenite aqueous solution per os to the experimental group animals also prevented the negative effect of the above amine on the processes of oxidative phosphorylation in the liver mitochondria (Table 6).

Thus, the respiration rate of rat liver mitochondria after adding succinate, the phosphorylation substrate - ADP and after its depletion, the oxidative phosphorylation disrupter - 2,4-DNP, and the phosphorylation rate and phosphorylation factor and respiratory control factor in animals in the experimental group, previously received aqueous sodium selenite solution and then poropylamines, were at the level of similar parameters of control group animals (Table 6. 6).

The obtained results confirm the previously expressed opinion about the protective effect of sodium selenite on the effect of lower aliphatic amines - ethyl and propylamine on oxidative phosphorylation in mitochondria, which can be used in the development of effective therapeutic and prophylactic agents caused by these toxicants.

Thus, on the basis of the studies on the effect of lower aliphatic amines, ethyl and propylamine, on the processes of oxidative phosphorylation in rat liver mitochondria, one important aspect of the mechanism of their toxic effects on the cell has been revealed, including oxidation of amines in cytoplasm with participation of oxygen and their conversion to proton, penetration of the latter through ion channels into mitochondria resulting in depolarization of mitochondrial membrane and hypoxia, decrease of ADP phosphorylation processes, decrease of macroergic compounds content. Activation of protective mechanisms in the liver involves intensification of reactions of metabolic transformations of amines in the cell, due to the action of 
TRADITIONAL MEDICINE AND PHARMACOLOGY. ACHIEVEMENTS, INNOVATIONS, AND ALTERNATIVES

aminooxygenases and several other enzymes, leading to the formation of non-toxic compounds from them.

Preliminary administration of sodium selenite to animals prevents the negative effects of ethyl- and propylamine on bioenergetic processes in mitochondria, which is associated with the participation of selenium in the antioxidant protection of body cells. 